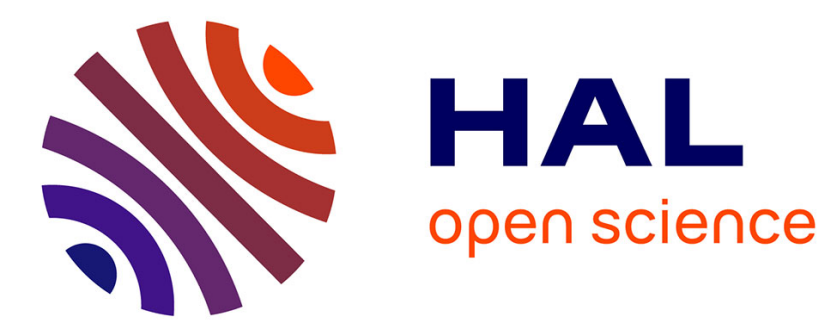

\title{
La photoémission
}

P. Vernier

\section{To cite this version:}

P. Vernier. La photoémission. Revue de Physique Appliquée, 1976, 11 (1), pp.23-30. 10.1051/rphysap:0197600110102300 . jpa-00244039

\section{HAL Id: jpa-00244039 https://hal.science/jpa-00244039}

Submitted on 1 Jan 1976

HAL is a multi-disciplinary open access archive for the deposit and dissemination of scientific research documents, whether they are published or not. The documents may come from teaching and research institutions in France or abroad, or from public or private research centers.
L'archive ouverte pluridisciplinaire HAL, est destinée au dépôt et à la diffusion de documents scientifiques de niveau recherche, publiés ou non, émanant des établissements d'enseignement et de recherche français ou étrangers, des laboratoires publics ou privés. 


\title{
LA PHOTOÉMISSION
}

\author{
P. VERNIER \\ Laboratoire de Photoélectricité de l'Université de Dijon, \\ Equipe de physique des Solides
}

\begin{abstract}
Résumé. - Le modèle à 3 étapes permet d'interpréter la distribution énergétique des photoélectrons et d'en tirer des informations sur la physicochimie des couches superficielles. On étudie les limitations de ce modèle et notamment celles qui résultent des effets de surface.

Le dernier paragraphe est consacré aux mesures simultanées de la distribution angulaire et énergétique des photoélectrons qui commence à apporter une information beaucoup plus riche que la simple distribution énergétique.
\end{abstract}

Abstract. - The 3 step model can explain the photoelectron energy distribution and is useful to extracting information about the surface layers. The limits of this model and especially those that result from the surface effects are discussed from both theoretical and experimental points of view.

The last paragraph deals with measurements of both angular and energy distributions of photoemitted electrons. This method is beginning to bring information which is much more precice than mere energy distributions.

1. Introduction. - L'objet de cette conférence est de préciser en pensant à des utilisateurs éventuels la nature des informations que la photoémission peut apporter à la connaissance des solides. Un physicochimiste demande à une analyse, la nature des espèces chimiques présentes, leur distribution dans le solide et les liaisons dans lesquelles les atomes sont engagés. Les données brutes de l'expérience sont les structures (pics, épaulements, points d'inflexion, minimums, seuils, etc...) que l'on peut observer dans la distribution spectrale du rendement quantique où la distribution énergétique des photoélectrons émis. Récemment on a pu associer à l'analyse énergétique des photoélectrons une analyse angulaire, obtenant ainsi des structures beaucoup plus nombreuses et riches en informations. Nous y consacrerons un paragraphe à la fin de cet exposé.

On pourrait se borner à réaliser des expériences sur des solides connus puis inconnus. En fait pour lever les ambiguités laissées par une démarche purement empirique autant que pour la satisfaction de l'esprit, un minimum d'interprétation théorique a été effectué à l'aide du modèle à 3 étapes notamment par Spicer et son école. Nous nous proposons d'abord d'en discuter les bases :

2. Le modèle à 3 étapes. - On suppose que la photoémission peut être séparée en 3 étapes indépendantes.

1) Absorption suivant un mécanisme qui est identique à celui que l'on observe dans la masse du solide.

(*) Associée au C. N. R. S. n 19.
2) Propagation d'un électron excité vers la surface du solide.

3) Traversée de la surface par cet électron.

On ajoute en général à cette division en étapes une analyse spatiale ce qui amène à l'expression classique du rendement quantique [1] :

$$
Y=\int D(z) p_{0} \mathrm{e}^{-z / L} \mathrm{~d} z
$$

avec

$$
D(z)=\frac{4 \pi n k}{\lambda \cos i_{0}} \frac{\left|E_{x}\right|^{2}+\left|E_{y}\right|^{2}+\left|E_{z}\right|^{2}}{\left|E_{0}\right|^{2}}
$$

$D(z) \mathrm{d} z$ est la probabilité pour un photon incident d'être absorbé à une distance de la surface comprise entre $z$ et $z+\mathrm{d} z$.

$E_{0}$ est l'amplitude complexe du champ électrique dans l'onde incidente (dans le vide).

$E_{x}, E_{y}$ et $E_{z}$ sont les amplitudes complexes des composantes du champ électrique suivant les 3 axes de référence dans le solide.

$n-i k$ est l'indice de réfraction du solide.

$i_{0}$ est l'angle d'incidence de la lumière (dans le vide).

La distribution énergétique $n(E)$ des photoélectrons émis (éventuellement dans un cône donné) peut être analysée de façon analogue :

$$
n(E)=\int D(z) p(E) \mathrm{e}^{-z / L(E)} \mathrm{d} z
$$

$p_{0} \mathrm{e}^{-z / L}\left(\right.$ ou $\left.p(E) \mathrm{e}^{-z / L(E)}\right)$ représente la probabilité pour que l'absorption d'un photon à la distance $z$ de la 
surface provoque l'émission d'un électron (d'énergie voisine de $E$ ). Nous pouvons interpréter l'exponentielle comme la probabilité pour un électron excité d'atteindre la surface et $p_{0}($ ou $p(E))$ comme la probabilité de traverser la surface. Mais en raison du large spectre des excitations produites par l'absorption d'un photon, $p_{0}$ et $L$ ne représentent que des valeurs moyennes.

On a envisagé d'autres formes que l'exponentielle $[2,3]$ mais pratiquement elles fournissent des résultats à peu près équivalents.

3. Validité du modèle à 3 étapes. - Ce modèle semble très naturel pour décrire les contributions au courant photoélectrique qui peuvent être associée à un niveau initial localisé dans le voisinage d'un atome : c'est le cas des niveaux profonds et plus généralement des bandes étroites. Les états électroniques des atomes isolés, moyennant une faible correction pour tenir compte des plus proches voisins représentent bien ces niveaux électroniques. Ils sont donc caractéristiques des espèces chimiques avec un faible déplacement qui renseigne sur les liaisons dans lesquels l'atome est engagé [4]. L'absorption fait passer un électron du niveau initial localisé à un niveau final d'énergie supérieure au niveau du vide et qui doit être décrit dans le solide parfait par une fonction de Bloch non localisée. Toutefois pour avoir l'énergie de cet état de Bloch on doit ajouter, au résultat déduit du théorème de Koopmans, l'énergie de relaxation positive qui est libérée par le réarrangement des états électroniques à la suite de l'apparition d'un trou localisé dans le voisinage de l'atome considéré. Il faudrait aussi tenir compte du fait qu'une partie de l'énergie du photon peut être prise par un autre électron de l'atome considéré pour l'amener dans un niveau supérieur du même atome (shake up) ou dans un état délocalisé (shake off). Les effets à $N$ corps ne peuvent être complètement décrits par une perturbation de la seule propagation de l'électron excité qui se traduit dans le modèle à 3 étapes par le facteur $\mathrm{e}^{-z / L}$.

Dans le paragraphe précédent nous n'avons pas considéré les effets de la superposition des sources de photoélectrons que constituent les différents atomes. En fait les ondes de Bloch au niveau excité qui résultent de ceux-ci sont synchronisés par l'onde électromagnétique qui leur a donné naissance, elles peuvent donc interférer. La règle de conservation du vecteur d'onde dans les transitions provient de ces interférences. Toutefois la diffusion qui résulte des différentes interactions subies par une onde de Bloch perturbe sa phase et par conséquent atténue les effets des interférences et de la règle de consarvation du vecteur d'onde qui en résulte. On pourrait envisager de définir une grandeur semi-empirique qui pourrait êtrel'homologue, en matière d'onde électronique du domaine de cohérence en matière d'onde électromagnétique. Ce domaine permettrait de définir quantitativement une loi intermédiaire entre les transitions directes définies par la règle de conservation du vecteur d'onde et les transitions non directes introduites par Spicer [5].

En fait dans le cas où le niveau initial appartient à une bande étroite, les distributions énergétiques que nous pouvons prévoir sont très voisines, que la règle de conservation du vecteur d'onde soit respectée ou non dans les transitions. La possibilité de localiser dans l'espace le niveau d'énergie profond d'un solide parfait résulte de la dégénérescence approchée par rapport au vecteur d'onde.

L'utilisation du modèle à 3 étapes pour l'émission à partir de niveaux d'une bande large semble a priori, plus difficile que dans le cas précédent. Si nous tentons de définir le niveau d'énergie initial d'un électron à partir d'un atome isolé, nous devrons faire intervenir l'interaction de voisins d'autant plus éloignés que la bande est plus large et que l'incertitude admise sur l'énergie est plus faible. Pour définir l'énergie initial d'un électron, il faut qu'il règne sur le point où il a été excité une incertitude qui peut atteindre l'ordre de grandeur de la profondeur d'extraction des électrons.

Nos conclusions rejoignent donc celles qui ont été tirées par Saint-James au congrès de Vittel, il y a 2 ans, à partir du formalisme de la réponse quadratique [6].

Nous pouvons répondre, en partie, à ces objections et maintenir une séparation du processus photoélectrique en une étape d'absorption et une étape de propagation si nous renonçons à localiser dans l'espace les états successifs occupés par l'électron avant et après l'absorption du photon. Il est alors possible d'utiliser avec une bonne approximation la règle d'or de Fermi pour décrire l'étape absorption à l'aide de matrices de transition entre des états monoélectroniques vacants et des états monoélectroniques excités. On définit ensuite la probabilité pour que l'état monoélectrique excité donne naissance à l'émission d'un électron. En fait, c'est ainsi que l'on justifie habituellement la règle de conservation du vecteur d'onde et que l'on introduit l'Energy Distribution of Joint Density of States (EDJDOS) [7].

Pour décrire correctement les zones proches de la surface les états monoélectroniques qui servent de base pour la représentation du solide ne doivent pas être les mêmes que pour un solide infini. Nous pouvons définir une couche de transition par les 2 caractéristiques suivantes :

1) Les fonctions d'ondes du solide infini (fonctions de Bloch pour un solide parfait) y sont modifiées d'autant plus que la surface est plus proche.

2) Des états dits de surface sont complètement localisés dans cette couche.

De ce fait l'absorption du solide semi-infini est différente de l'absorption du solide infini.

Dans certains cas il faut tenir compte, dans le traitement de l'absorption optique, des interactions des états monoélectroniques entre eux ou avec d'autres excitations. Les effets de relaxation, de shake up et de shake 
off dans le domaine $\mathrm{X}$ et UV lointain en sont des exemples, les transitions indirectes avec émission ou absorption de phonons dans le domaine visible et infrarouge en sont un autre.

En outre la probabilité de transition doit en toute rigueur tenir compte de toute l'évolution du solide à la suite de l'absorption du photon. L'élargissement du niveau final par suite de sa faible durée de vie donne une idée semi-classique de ce problème qui ressort de la théorie à $N$ corps. Malheureusement l'étude de l'interaction électron-électron qui est la principale limite de la durée de vie de l'état électronique excité et de la probabilité pour que cet état donne naissance à l'émission d'un électron, n'a jusqu'à présent pu être abordée que sur un plan qualitatif.

On a introduit dans les études d'absorption la notion d'excitation élémentaire du solide (plasmon, exciton, etc...). Pour les utiliser en photoémission il faut déterminer la probabilité qu'elles ont de se décomposer en provoquant l'émission d'un électron. Endriz [8] a pu calculer cette probabilité pour un plasmon de surface.

4. Définition de l'effet de surface. - Les structures que l'on observe ne renseignent que sur les couches superficielles en raison des interactions subies par les électrons dans les états excités (essentiellement en raison de l'interaction électron-électron). Pour étudier ces interactions et pour localiser les espèces chimiques détectées à partir des données photoélectriques, nous pouvons garder le modèle à 3 étapes localisées et analyser les résultats expérimentaux à l'aide des expressions (1) et (2). Cette démarche revient en fait à substituer au solide réel un système homogène à l'intérieur de sa surface et défini par des paramètres macroscopiques $n-i k, L$ et $L(E)$, qui sont uniformes jusqu'à la surface du solide où ils présentent une discontinuité.

Tous ces paramètres peuvent servir d'intermédiaires pour une discussion plus approfondie des mécanismes à l'échelle atomique. Ce modèle de solide homogène et discontinu à la surface conduit pour la réflexion de la lumière aux formules de Fresnel qui, à notre connaissance n'ont jamais été mises en défaut expérimentalement dans le cas des surfaces planes sans rugosité pour lesquelles elles ont été démontrées [9]. Le caractère macroscopique de ce modèle ne constitue pas une approximation dans la description d'un élément de volume éloigné de la surface. Si les paramètres $n-i k$, $p_{0}$ et $L$ ne sont pas déterminés par une méthode approchée, tout écart entre le résultat de ce modèle à 3 étapes et les faits expérimentaux pourra être attribué aux perturbations apportées par la présence de la surface. Nous pouvons donc définir l'effet de volume par les formules (1), (2) et (3). Nous définirons alors le rendement photoélectrique de surface $Y_{\mathrm{s}}$ comme la différence entre le rendement expérimental $Y_{\text {exp }}$ et le rendement de volume.

$$
Y_{\mathrm{s}}=Y_{\text {exp }}-\int D(z) p_{0} \mathrm{e}^{-z / L} \mathrm{~d} z
$$

On peut définir de même l'effet de la surface sur la distribution énergétique des photoélectrons.

$$
n_{\mathrm{s}}(E)=n_{\exp }(E)-\int D(z) p(E) \mathrm{e}^{-z / L(E)} \mathrm{d} z
$$

$Y_{\mathrm{s}}$ et $n_{\mathrm{s}}(E)$ résultent de la modification apportée à l'ensemble des fonctions d'onde monoélectroniques et de leurs interactions introduites dans toute la couche de transition par la présence de la surface. Ils peuvent être $a$ priori aussi bien positifs que négatifs. Dans la pratique on peut cependant citer des contributions à l'effet de surface qui sont nécessairement positives et s'ajoutent à l'effet de volume sans interférence.

5. Les effets de surface dus à des niveaux de surface. - $a$ ) De nombreux auteurs (par exemple Wagner et Spicer, Eastman et Grobman [10]) attribuent certaines structures de la courbe de distribution énergétique des photoélectrons, à des transitions à partir d'un niveau de surface intrinsèque ou non (Fig. 1).

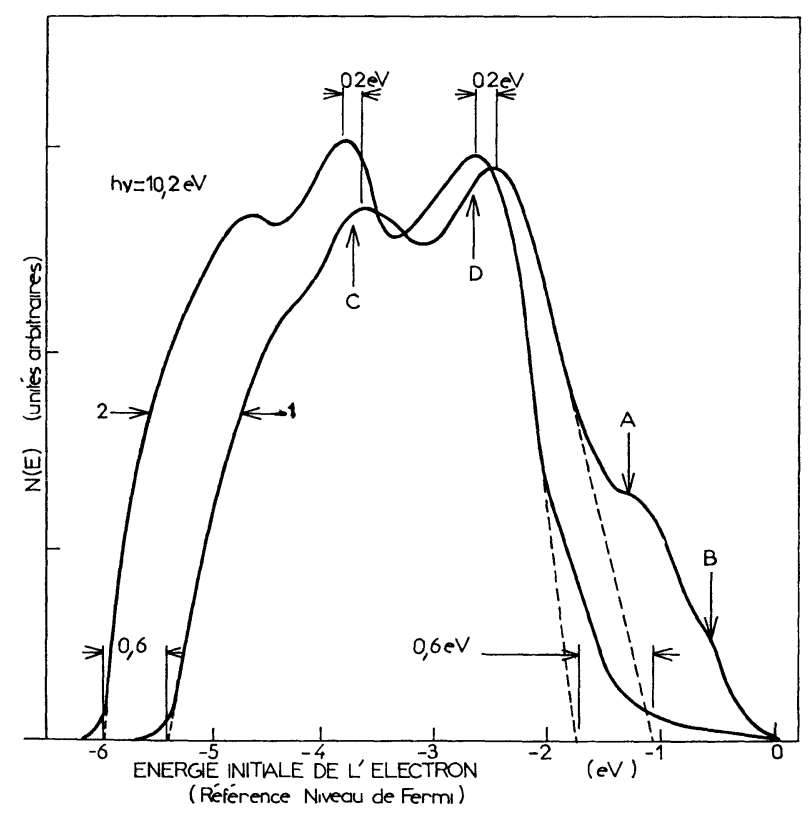

Fig. 1. - Distribution énergétique des photoélectrons émis par une face clivée de silicium avant et après exposition à l'oxygène. (D'après Wagner et Spicer [5]).

L'un des arguments décisifs en faveur de l'origine superficielle de ces structures est leur sensibilité à la pollution de la surface.

b) Certains niveaux électroniques supérieurs au niveau du vide peuvent être associés à un vecteur d'onde complexe du côté du solide (fonction d'onde évanescente) et réel du côté du vide (fonction d'onde progressive). Feuerbacher [11] a expliqué certaines structures observées dans la distribution énergétique des électrons émis normalement à la face (110) du tungstène (Fig. 2), par une transition entre un niveau occupé du solide et un tel niveau ; parce qu'aucun état 
ne peut être associé à un vecteur d'onde normal à la surface pour les énergies finales de l'électron impliquées par les structures 4, 5, 6 (Fig. 2 et 3).

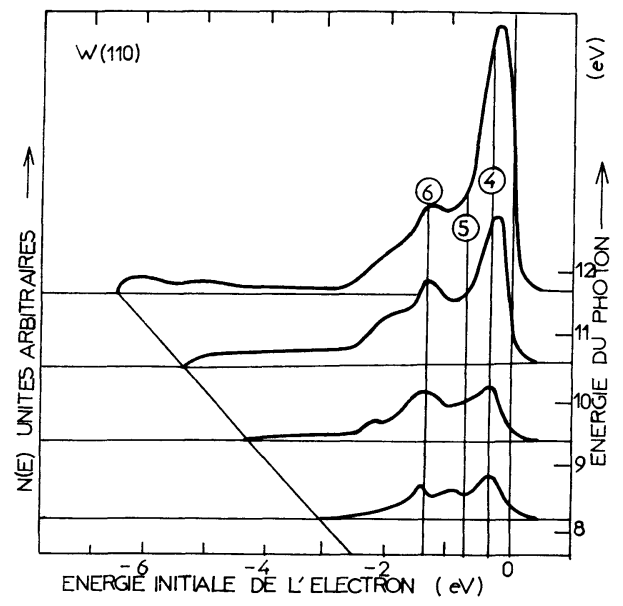

Fig. 2. - Distribution énergétique des photoélectrons émis normalement par la face (110) du tungstène pour des photons dont l'énergie varie de 7,7 eV à 11,7 eV. (D'après Feuerbacher et Christensen [11]).

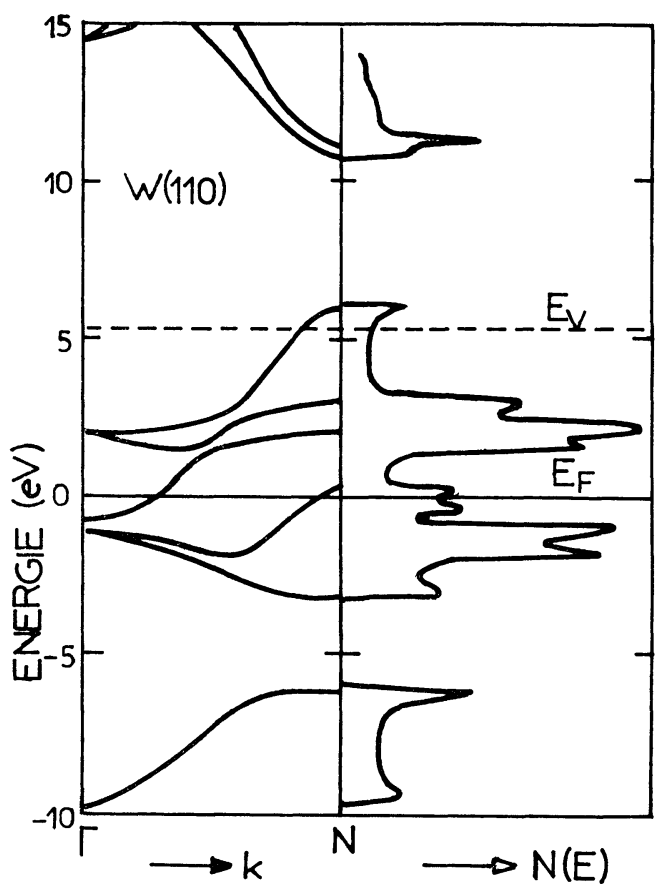

FIg. 3. - Structure de bande du tungstène et densité d'états pour lesquels le vecteur d'onde est parallèle à la direction (110). (D'après Feuerbacher et Christensen).

6. L'émission de surface due à la réflexion des électrons sur les barrières de surface. - Dans un gaz d'électrons libres il n'y a pas d'effet de volume puisque $n k=0$ (voir formule 2). L'absorption de la lumière et la photoémission résultent alors uniquement de la réflexion des électrons sur la surface.

Tous les développements théoriques aussi bien les plus anciens de Makinson [12] ou Mitchell [13] que ceux plus récents d'Adawi [14], Mahan [15], Schaich et Ashcroft [16], Caroli et al. [6], s'accordent pour prévoir que seule la composante du champ électrique normale à la surface peut alors provoquer ce type d'émission photoélectrique.

Dans un métal réel, la réflexion des électrons de Bloch sur la surface provoque un effet de surface qui se compose avec l'effet de volume suivant une loi complexe, avec des interférences quantiques qui peuvent $a$ priori, être aussi bien constructives que destructives $[15,16]$. En outre, l'interaction des électrons de Bloch avec la surface est plus complexe qu'une simple réflexion spéculaire parce que la structure périodique des faces cristallines idéales et la structure irrégulière des surfaces réelles produit toujours une diffraction ou une diffusion des électrons. Dans une première approximation nous pouvons admettre avec Endriz [8] que pour une surface lisse la réflexion des électrons de Bloch produit un effet de surface qui s'ajoute sans interférence à un effet de volume décrit par les éq. (1), (2) et (3).

Nous pouvons aussi admettre que, comme pour des électrons libres, le rendement photoélectrique qui est dû à la réflexion des électrons est proportionnel au carré du module de l'amplitude de la composante normale du champ électrique, dans le solide, dans le voisinage de la surface. Pour des raisons de commodité nous pouvons écrire :

$$
Y_{\mathrm{s}}=K_{\mathrm{s}} D_{\mathrm{z}}(0)
$$

avec

$$
D_{z}\left(0^{+}\right)=\frac{4 \pi n k}{\lambda \cos i_{0}} \frac{\left|E_{z}\right|^{2}}{\left|E_{0}\right|^{2}}
$$

bien que l'indice de réfraction $n-i k$ ne soit pas lié de façon directe à l'absorption de surface.

Endriz [8], Callcott et Arakawa [17] et plusieurs chercheurs du laboratoire de Photoélectricité de Dijon [18], sont partis de ces bases pour déterminer la contribution de l'effet de surface en comparant les rendements photoélectriques de métaux simples obtenus pour les 2 polarisations de la lumière et pour divers angles d'incidence.

Nous montrerons ici quelques résultats tirés du travail de Goudonnet et al. [18] sur des couches minces d'argent. La figure 4 montre la profondeur d'extraction des photoélectrons tirée des mesures photoélectriques effectuées en polarisation $s$. On peut remarquer la concordance des résultats obtenus pour les divers angles d'incidence. Il est important dans cette mesure que la couche ne présente aucune rugosité, afin que la lumière de polarisation $s$ ne produise aucun effet de surface. Nous renverrons sur ce point à l'article qui sera publié. La figure 5 montre le rapport des rendements photoélectriques de surface $Y_{\mathrm{s}}$ et de volume $Y_{v_{p}}$ obtenus avec une lumière polarisée $p$.

Le pic observé dans le voisinage de la fréquence de plasma vers 3,8 eV est dû à la distribution de l'énergie électromagnétique entre la composante tangentielle du 


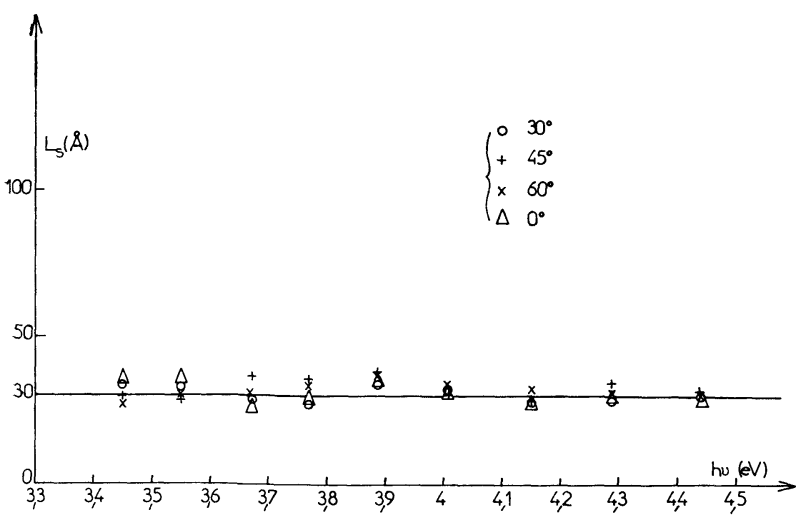

Fig. 4. - Profondeur d'extraction des photoélectrons pour une couche d'argent (d'épaisseur $280 \AA$ ), déduite des mesures photoélectriques faites sous incidence de $0^{\circ}, \times 30^{\circ}, 45^{\circ},+60^{\circ}$. (D'après Goudonnet, Chabrier et Vernier [18]).

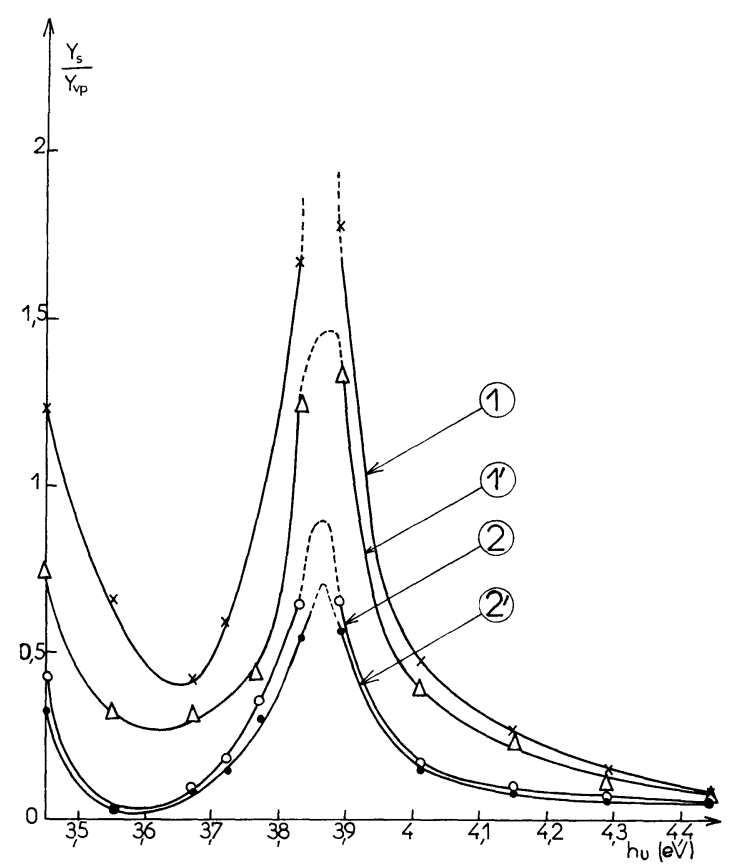

Fig. 5. - Rapport des rendements photoélectriques associés à un effet de surface et à un effet de volume pour une couche .mince d'argent d'épaisseur $280 \AA$ éclairée à travers le substrat (1 et 2 ) et directement du côté du vide ( $\left(1^{\prime}\right.$ et $\left.2^{\prime}\right)$. L'angle d'incidence de la lumière de polarisation $p$ était $30^{\circ}$ pour 2 et $2^{\prime}, 60^{\circ}$ pour 1 et $1^{\prime}$. (D'après Goudonnet, Chabrier et Vernier [18]).

champ électrique et la composante normale, c'est-àdire au facteur :

$$
\frac{D_{z}(0)}{\int D(z) \mathrm{e}^{-z / L} \mathrm{~d} z}
$$

La grandeur $K / p_{0}$, homogène à une longueur permet de comparer les effets de surface et de volume produits par une même amplitude du champ électromagnétique. On peut remarquer sur la figure 6 , la croissance monotone de ce facteur que nous pouvons attribuer à la proximité du seuil pour l'effet de volume.

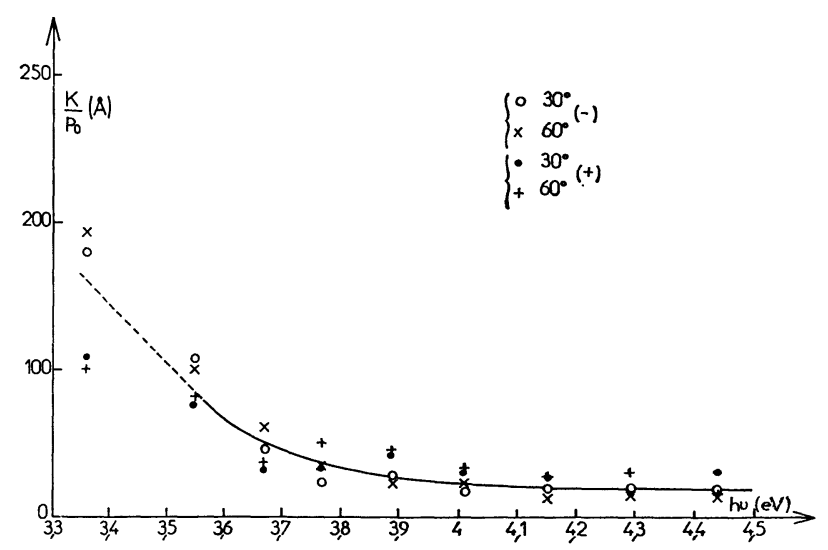

FIG. 6. - Variation de la grandeur $K_{\varepsilon} / p_{0}$ caractéristique de la photoexcitation par effet de surface avec l'énergie des photons. (D'après Goudonnet, Chabrier et Vernier [18]).

Il faut remarquer que l'exemple de l'argent a été choisi précisément parce qu'il favorise l'effet photoélectrique de surface, à la fois à cause de la proximité du seuil pour l'effet de volume et à cause de ses propriétés optiques. Dans la grande majorité des cas, l'effet de surface sera petit et la théorie à 3 étapes rendra bien compte de l'émission photoélectrique. Cette étude expérimentale confirme donc les travaux théoriques de Feibelman et Eastman [19].

7. Analyse angulaire et énergétique des photoélectrons. - Le rendement quantique global pour une lumière monochromatique ou même la distribution énergétique des électrons émis dans un angle solide de $2 \pi$ résultent de la superposition d'un très grand nombre de processus élémentaires. Cela facilite les interprétations théoriques en permettant dans un grand nombre de cas d'obtenir le courant photoélectrique total par l'addition des courants photoélectriques qui résulteraient de chacun des processus, s'il était seul. Une approximation de phase aléatoire permet de négliger les interférences quantiques.

En revanche cette superposition laisse des ambiguïtés par exemple nous avons vu que de telles données expérimentales ne permettent pas de trancher entre les processus de transition directe et non directe. Ces ambiguïtés devraient disparaître en grande partie si on combinait à l'analyse énergétique une analyse angulaire. Notons que l'approximation de la phase aléatoire pourrait cesser d'être valable et que les différents processus élémentaires pourraient cesser de composer leurs effets par simple addition.

L'appareillage devient très complexe parce qu'il doit fonctionner sous ultra-vide et doit comporter des transmissions mécaniques pour balayer toutes les directions d'émission plus éventuellement des transmissions pour changer l'orientation de l'échantillon. En outre un contrôle de l'état de la surface par diffraction d'électrons lents et analyse Auger apporte souvent un complément essentiel sinon indispensable.

Feuerbacher et ses collaborateurs [11] se bornent à 
déterminer la distribution énergétique des électrons émis normalement à la surface à l'aide d'un analyseur cylindrique électrostatique à $127^{\circ}$. Le fait de sélectionner les électrons émis dans cette direction apporte déjà une donnée nouvelle en limitant le nombre de processus élémentaires qui interviennent. La figure 7 montre les distributions énergétiques pour les faces (100) et (111) qui sont très différentes de celles qu'il a observées pour la face (110) (Fig. 3). Une interprétation détaillée de chaque structure a été donnée par Feuerbacher et Christensen à partir du modèle à étapes.
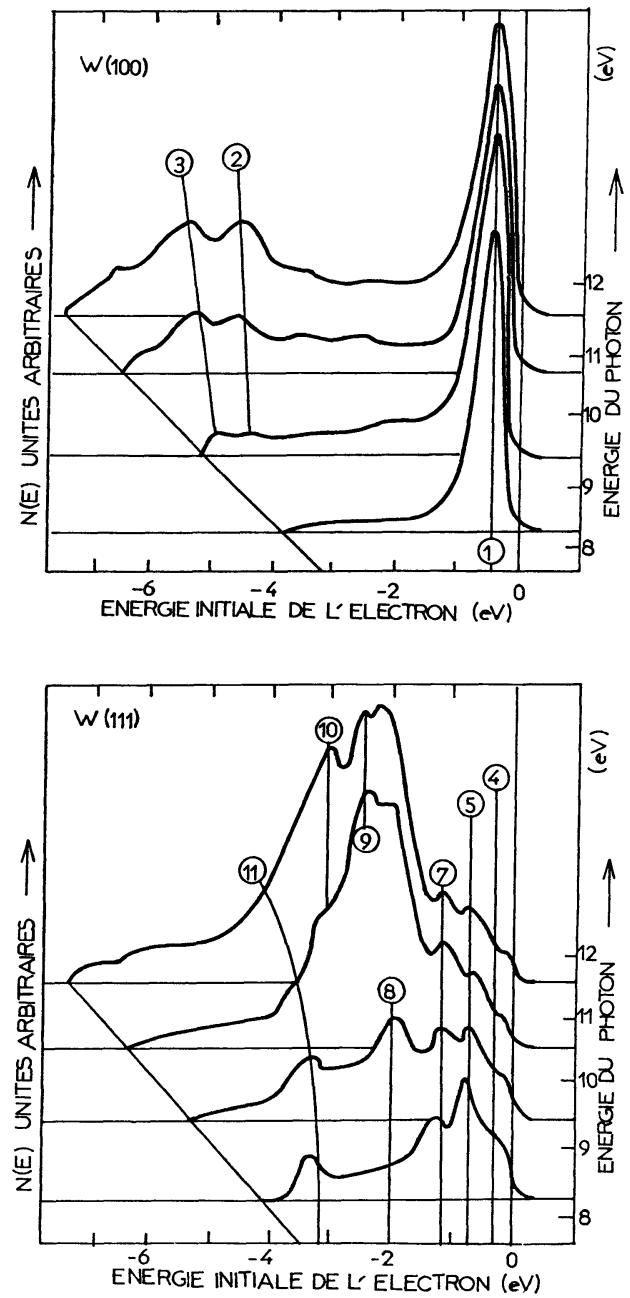

Fig. 7. - Distribution énergétique des photoélectrons émis normalement par les faces (111) et (100) du tungstène pour des photons dont l'énergie varie de $7,7 \mathrm{eV}$ à $11,7 \mathrm{eV}$. (D'après Feuerbacher et Christensen [11]).

Plusieurs dispositifs expérimentaux sont maintenant en œuvre pour fournir une analyse énergétique des électrons émis dans chaque direction.

L'étude des monocristaux de tungstène a été reprise par Callcott et Turtle [20] à l'aide d'un dispositif à potentiel retardateur qui fournit une analyse énergétique des électrons émis dans chaque direction. Ils ont confirmé dans l'ensemble les résultats de Feuerbacher. Mais nous n'avons pas la place ici de donner une interprétation détaillée de chaque structure observée.
Smith et Traum ont mesuré, aussi, avec un dispositif à potentiel retardateur, la distribution énergétique des électrons émis, dans toutes les directions par la face de clivage de composés lamellaires $\mathrm{TaSe}_{2}$ et $\mathrm{TaS}_{2}$. L'intérêt de ces composés est que en première approximation l'énergie des électrons dans le solide, ne dépend que de la composante tangentielle du vecteur d'onde $\mathbf{k}_{\|}$. Dans un schéma à étapes, la composante tangentielle de la vitesse de l'électron émis est $\hbar k_{\|} / m$. Les données expérimentales de Smith et Traum peuvent donc être directement comparées aux résultats des calculs de la structure de bande.

La figure 8 est un diagramme polaire obtenu pour une face de clivage de $\mathrm{TaSe}_{2}$ (de structure $1 \mathrm{~T}$ ) et des

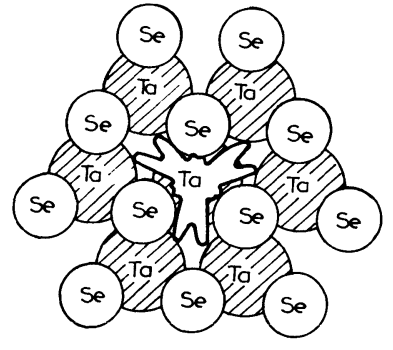

(a)

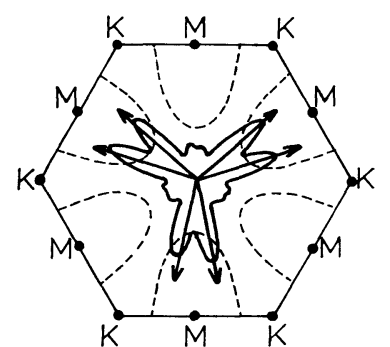

(b)
Fig. 8. - Diagramme polaire montrant les variations du nombre d'électrons $d$ émis sous l'action de photon d'énergie $10,2 \mathrm{eV}$, à partir du niveau de Ta par la face de clivage de $\mathrm{TaSe}_{2}$ (de structure $1 \mathrm{~T}$ ) dans des directions faisant avec la normale un angle de $55^{\circ}$. Le diagramme est superposé sur la figure $8 a$ à une représentation de 2 couches atomiques externes et sur la figure $8 b$ à la coupe de la surface de Fermi (en tirés) par le plan $k_{z}=0$. Les flèches représentent les composantes tangentielles du vecteur $k$ associées aux transitions directes. (D'après Smith et Traum [2]).

photons d'énergie 10,2 eV. On a représenté, en fonction de l'azimuth, les variations du courant photoélectrique transporté par les électrons émis, à partir de la couche $\mathrm{d} d u$ tantale, dans une direction faisant un angle de $55^{\circ}$ avec la normale. Ce diagramme est superposé en $8 a$ à la structure des 2 couches atomiques externes et en $8 b$ à la coupe de la surface de Fermi par le plan $k_{z}=0$. Les flèches représentent les composantes tangentielles du vecteur $\mathbf{k}$ compatibles avec des transitions directes. On peut noter que leurs directions coïncident avec celles des maximums d'émission.

Nous devrions encore citer plusieurs publications sur les effets angulaires en photoémission [22]. Nous nous bornerons à une expérience en cours ici à Dijon [23].

En combinant la dispersion angulaire à la dispersion énergétique, on allonge considérablement la durée des expériences à la fois parce que la quantité de données à acquérir est accrue et parce que le nombre de photons disponibles pour chaque donnée est réduit. Pour pallier cet inconvénient F. Pauty enregistre le spectre à 2 dimensions produit par un système dispersif à l'aide d'une caméra électronique, sur une plaque électronographique (Fig. 9). 


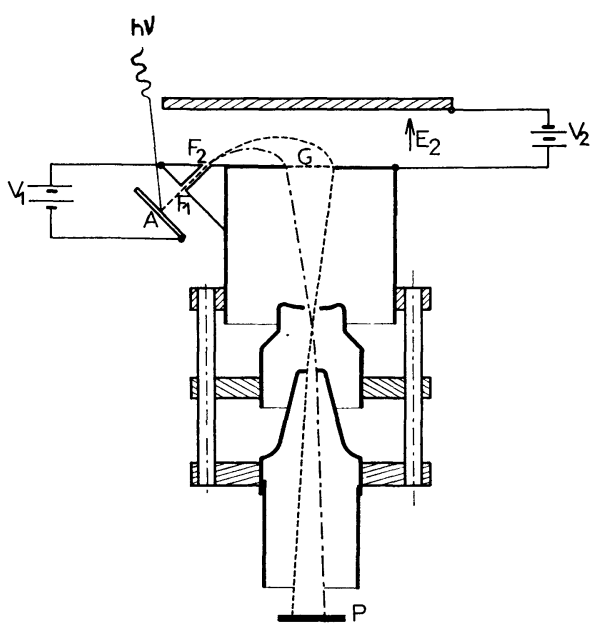

Fig. 9. - Dispositif analyseur pour photoélectrons de F. Pauty. Les électrons émis par le point $\mathrm{A}$ dans le plan défini par les fentes $F_{1}$ et $F_{2}$ sont dispersés par le champ uniforme $E_{2}$. Sur la grille $\mathrm{G}$ se forme un spectre à 2 dimensions. Parallèlement au plan de la figure les électrons sont dispersés selon leur énergie, perpendiculairement au plan de la figure selon leur direction d'émission. Les électrons qui traversent la grille $G$ sont accélérés et focalisés sur une plaque photographique qui les enregistre.

(D'après F. Pauty, Matula et Vernier [23]).

La figure 10 montre le diagramme polaire des électrons émis par une couche d'or polycristalline à diverses énergies. On peut noter les structures très fines et riches

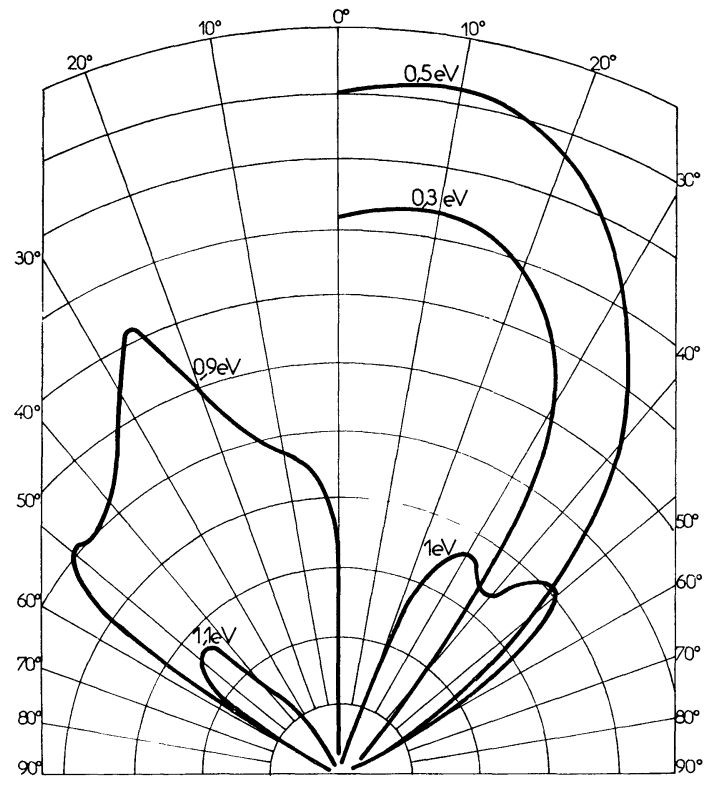

Fig. 10. - Diagramme polaire montrant la distribution des photoélectrons émis par une couche d'or polycristalline avec différentes énergies cinétiques en fonction de l'angle que fait la direction d'émission avec la normale à l'échantillon. Comme cette direction est un axe de symétrie nous n'avons représenté que la moitié du diagramme. (D'après Pauty, Matula et Vernier [23]) d'information que l'on observe déjà avec une énergie de photon aussi faible que $4,87 \mathrm{eV}$.

8. Conclusion. - L'analyse angulaire donne aujourd'hui une et même deux nouvelles dimensions à la photoémission comme méthode d'analyse puisque la direction d'émission est caractérisée par 2 angles. Déjà pour des photons du proche ultra-violet elle fait apparaître de nombreuses structures (Fig. 10) et c'est toute la structure de bande qui a pu être révélée sans ambiguité par Smith et Traum dans leur étude des composés lamellaires. La limitation principale de cette méthode viendra sans doute de la diffusion des électrons excités qui brouillera l'image espérée de la structure électronique du solide. Dans le cas d'une analyse purement énergétique, les interactions électron-électron gênent souvent peu, parce que les électrons secondaires sont rejetés dans des parties du spectre éloignées des structures intéressantes, les interactions électron-phonon ne produisent qu'un élargissement en général faible des structures. Dans une analyse angulaire une interaction électron-phonon importante risque de brouiller complètement l'information angulaire. C'est probablement pour cette raison que les structures observées dans la figure 10 sont beaucoup plus fines pour les électrons d'énergie maximale que pour les autres.

Du fait de la richesse des informations apportées, l'analyse angulaire des photoélectrons risque aussi de faire apparaître l'insuffisance des modèles théoriques utilisés. Nous avons vu que la superposition des contributions d'un grand nombre de processus élémentaires peut être traitée par la simple addition de leurs effets. Il peut n'en plus être de même si le nombre devient petit comme ce sera le cas avec une analyse à la fois angulaire et énergétique et des interférences quantiques auront alors beaucoup plus de chances de se manifester. En outre les défauts du modèle dans la description de chaque processus élémentaire ne pourront plus se compenser.

La difficulté majeure des développements théoriques futurs sera probablement la diffusion électron-électron. C'est principalement à cause de cette interaction que nous ne pouvons tirer de la photoémission que des renseignements sur des couches proches de la surface. Cet effet d'écran est décrit de façon satisfaisante dans le processus à 3 étapes par la profondeur d'extraction. Mais l'interaction électron-électron a beaucoup d'autres conséquences que l'on ne sait traiter qu'avec des modèles très simplifiés même dans le problème relativement simple de l'absorption de la lumière par un solide infini. Dans l'absorption par un solide semi-infini et la photoémission, la conjonction de l'interaction électron-électron et des perturbations introduites par la surface, constitue un problème beaucoup plus complexe. 


\section{Bibliographie}

[1] Voir par exemple :

Vernier, P. J., Goudonnet, J. P., Chabrier, G., CorNAZ, J., J. Opt. Soc. Amer. 61 (1971) 1065, ou :

Coquet, E. et Vernier, P., C. R. Hebd. Séan Acad. Sci. 262 (1966) 1141.

[2] Vernier, P. J., Progress in Optics, vol. 14 (à paraître).

[3] Hirschberg, K. et Deutscher, K., Phys. Stat. Sol. 26 (1968) 527 ;

Hofmann, H. H. et Deutscher, K., Z. Phys. 236 (1970) 288.

[4] Siegbahn, K., J. Electron Spectrosc. Relat. Phenom. 5 (1974) 3.

Voir aussi les autres articles du même numéro consacrés à la conférence internationale sur la spectroscopie des électrons, tenue à Namur en 1974.

[5] Berglund, C. N. et Sicer, W. E., Phys. Rev. 136 (1964) A 1030.

SPICER, W. E., Phys. Rev. 154 (1967) 385.

[6] Caroli, C., Lederer-Rozenblatt, D., Roulet, B., Saint-James, D., Phys. Rev. B 4 (1973) 4552.

[7] Voir, par exemple :

Harbeke, G., Optical properties of Semiconductors édité par F. Abeles (North Holland) 1972, p. 21.

STERN, F., «Elementary theory of the optical properties of solids » Solid State Physics 15.

[8] ENdriz, J. G. et SPICER, W., Phys. Rev. Lett. 24 (1970) 64; Endriz, J. G., Phys. Rev. B 7 (1973) 3463 ;

Flodstrom, S. A. et Endriz, J. G., Phys. Rev. Lett. 31 (1973) 893.

[9] Vernier, P. J., Acta electron. 16 (1973) 181.

[10] Wagner, L. F. et Spicer, W. E., Phys. Rev. Lett. 28 (1972) 1381.

Eastman, D. E. et Grobman, W. D., Phys. Rev. Lett. 28 (1972) 1378.
[11] Feuerbacher, B. et Fitton, B., Phys. Rev. Lett. 30 (1973) 923.

Christensen, E. et Feuerbacher, B., Phys. Rev. B 10 (1974) 2349.

[12] Makinson, R. E. B., Phys. Rev. 75 (1949) 1909.

[13] Mitchell, K., Proc. R. Soc. A 146 (1934) 442 et A 153 (1936) 513.

[14] Adawi, I., Phys. Rev. 134A (1964) 788.

[15] Mahan, G. D., Phys. Rev. B 2 (1970) 4334.

[16] Schaich, W. L. et Aschcroft, N. W., Phys. Rev. B 3 (1970) 2452.

[17] Callcott, T. E. et Arakawa, E. T., Phys. Rev. B 11 (1973) 2750.

[18] Goudonnet, J. P., Chabrier, G. et Vernier, P., à paraître.

[19] Feibelman, P. J. et Eastman, D. E., Phys. Rev. B 10 (1974) 4932.

[20] Turtle, R. et Callcott, T. A., Phys. Rev. Lett. 34 (1975) 86.

[21] Smith, N. V. et Traum, M. M., Phys. Rev. B 11 (1975) 2087.

[22] Gerhardt, U. et Dietz, E., Phys. Rev. Lett. 26 (1971) 1477 ; Gustavssonn, T., Nilsson, P. O. et Wallden, L., Phys. Lett. A 37 (1971) 121.

Wooten, F., Huen, T., Windsor, H. V., Phys. Lett. A 36 (1971) 351.

Lindau, L. et Hagström, S. B. M., J. Phys. E 4 (1971) 936.

Fadley, C. S. et Bergström, S. A. L., Proc. Int. Conf. 1971, Electron Spectroscopy publié par D. A. Shirley (North Holland) p. 233.

Koyama, R. Y. et Hughey, L. R., Phys. Rev. Lett. 29 (1972) 1518.

Williams, R. H., Thomas, J. M., Burker, M. et Alford, N., Chem. Phys. Lett. 17 (1972) 142.

Poole, R. T., Leckey, R. C. G., Jenkin, J. G. et LiesenGANG, J., J. Electron. Spectrosc. 1 (1973) 371.

[23] Pauty, F., Matula, G. et Vernier, P. J., Rev. Sci. Instrum. 45 (1974) 1203. 